Orange Journal / Volumen 3 Número 5/ Enero - junio 2021

DOI: https://doi.org/10.46502/issn.2710-995X/2021.5.04

González González, A., Rivas Santana, M., Quiza Sardiñas, R., Paz Estévez, E.A., \& Pla Pérez, A. (2021).

Modelación de andamios porosos basados en las estructuras triplemente periódicas P y G. Orange Journal, 3(5), 30-41. https://doi.org/10.46502/issn.2710-995X/2021.5.04

\title{
Modelación de andamios porosos basados en las estructuras triplemente periódicas P y G
}

\section{Modeling of porous scaffolds based on the triply periodic structures $P$ and $G$}

Recibido: 22 de julio de 2021

Aceptado: 10 de septiembre de 2021

Escrito por:

Alejandro González González ${ }^{10}$

https://orcid.org/0000-0003-2904-9660

Marcelino Rivas Santana ${ }^{11}$

https://orcid.org/0000-0002-0305-515X

Ramón Quiza Sardiñas ${ }^{12}$

https://orcid.org/0000-0003-1293-6044

Elsa Adelina Paz Estévez ${ }^{13}$

https://orcid.org/0000-0002-2569-1747

Amalia Pla Pérez ${ }^{14}$

https://orcid.org/0000-0002-3048-6481

\section{Resumen}

En este trabajo se estudia la modelación de estructuras híbridas de andamios porosos para regeneración de tejido óseo basadas en las superficies minimales triplemente periódicas Giroide (G) y primitiva de Schwarz (P). El diseño de las probetas prismáticas híbridas, con dimensiones según la norma ASTM D695_15, se logra a partir de las ecuaciones que definen a cada estructura utilizando la función de enlace sigmoidea con valor $\mathrm{k}=0.5$ mediante el software CAS Wolfram Mathematica v11.2. Los aspectos relacionados con el uso de Mathematica como herramienta para el diseño de las probetas son discutidos en detalle. Las constantes de la ecuación de cada estructura son utilizadas como variables en un diseño factorial 32 para estudiar su efecto en la porosidad y tamaño de poros. A partir de regresión multilineal se obtienen las ecuaciones que relacionan los factores con las variables dependientes y se discuten los modelos obtenidos. Se concluye que el modelo bilineal es adecuado para la descripción de las variables de respuesta lo que justifica la elección del diseño experimental utilizado.

Palabras claves: Andamios porosos híbridos, superficies minimales triplemente periódicas, Mathematica, regresión multilineal.

\footnotetext{
Abstract

${ }^{10}$ Departamento de Mecánica, Universidad de Matanzas, Cuba.

${ }^{11}$ Centro de Estudios de Fabricación Avanzada y Sostenible (CEFAS), Universidad de Matanzas, Cuba.

${ }^{12}$ Centro de Estudios de Fabricación Avanzada y Sostenible (CEFAS), Universidad de Matanzas, Cuba.

${ }^{13}$ Centro de Estudios de Fabricación Avanzada y Sostenible (CEFAS), Universidad de Matanzas, Cuba.

${ }^{14}$ Universidad de Matanzas, Cuba.
}

In this work we study the modeling of hybrid porous scaffold structures for bone tissue regeneration based on the triply periodic minimal surfaces Gyroid (G) and Schwarz's primitive (P). The design of hybrid prismatic probes, with dimensions according to the norm ASTM D695_15, is made from the equations defining each of the structures using the sigmoid function with $\mathrm{k}=0.5$ within the CAS software Wolfram Mathematica $\mathrm{v}$ 11.2. The issues related to the use of Mathematica as a tool for the design of probes are discussed in detail. The constants within each structure are taken as factors in a 22 factorial design in order 
to study its effect on porosity and pore size. The model equations relating dependent variables with factors are obtained from multilinear regression analysis and discussed. It is concluded that a bilinear model is adequate to describe the response variables what justifies the factorial design chosen.

Keywords: Hybrid porous scaffold, triply periodic minimal surfaces, Mathematica, multilinear regression.

\section{Introducción}

Los andamios son estructuras de soporte sólidas utilizadas en injerto de tejidos y que tienen varios requisitos relacionados con sus propiedades mecánicas, biológicas y físico-químicas que les dan la capacidad para facilitar la creación de tejidos. Para diseñar andamios para regeneración de tejido óseo hay que tener en cuenta varios factores como (a) que tengan una red totalmente interconectada de poros que favorezca el transporte de sustancias y nutrientes (Nikolova \& Chavali, 2019), (b) que sean biocompatibles y biodegradables, (c) que tengan las propiedades mecánicas apropiadas como la rigidez y la resistencia mecánica teniendo en cuenta el uso que se desee dar al producto (Kanczler et al., 2020) y (d) que la morfología interna de los poros permita el crecimiento celular y la formación de nuevo tejido (Knychala et al., 2013; Zadpoor, 2014).

Existen diversos métodos para diseñar estructuras de andamios porosos. Entre ellos tenemos los métodos CAD para generar la celda unitaria a partir de sólidos elementales (Ahmadi et al., 2015; Naing, Chua, Leong, \& Wang, 2005), el método de intersección de imágenes binarias (Hollister, 2005; Saito et al., 2010; Wu, Spanou, Diez-Escudero, \& Persson, 2019), la modelación de la trayectoria de entramado en el método de fabricación por filamento fundido o deposición fundida (FDM) (Amirkhani, Bagheri, \& Zehtab Yazdi, 2012; Enrique Cuan-Urquizo et al., 2019; E. Cuan-Urquizo, Yang, \& Bhaskar, 2015; Giannitelli, Accoto, Trombetta, \& Rainer, 2014; Lee et al., 2012; Malinauskas et al., 2015; Moroni, de Wijn, \& van Blitterswijk, 2006; Norato \& Johnson, 2011) y el método de superficies implícitas que permite diseñar estructuras celulares con alto grado de complejidad (Gandy, Bardhan, Mackay, \& Klinowski, 2001) como las superficies minimales triplemente periódicas (SMTP).

Se denominan superficies minimales o extremales mínimas a las que satisfacen el problema de cálculo de variaciones de hallar la superficie orientable cerrada de menor área con frontera limitada por una curva cerrada espacial (Meeks III, 1990). Aunque existen muchas de estas superficies, las más estudiadas por lo interesantes de sus propiedades y de mayores aplicaciones técnicas, son las SMTP que son periódicas en las tres direcciones espaciales independientes, no se autointersecan y dividen al espacio tridimensional donde ellas están en dos sub-volúmenes conexos que no se interpenetran entre sí y que se denominan laberintos (D. Anderson, Davis, Nitsche, \& Scriven, 1990). El método implícito de diseño computacional de estas superficies se basa en el truncamiento del desarrollo de Fourier de la superficie que tiene la periodicidad espacial de alguna red cristalina (Gandy et al., 2001), lo que resulta en una ecuación implícita para cada estructura, con un grupo de parámetros que permiten tener mayor control del tamaño de poros y la porosidad. Entre las superficies más conocidas se encuentran la Giroide $(\mathrm{G})$ y la Primitiva de Schwarz (P), ambas con simetría BCC.

El interés por este tipo de superficies matemáticas viene de dos direcciones diferentes pues por un lado se ha descubierto la existencia de las SMTP en la naturaleza (Han \& Che, 2018) y por otro lado se ha publicado que la estructura del hueso trabecular presenta una curvatura promedio casi nula, que es una propiedad distintiva de las SMTP (Bidan, Wang, \& Dunlop, 2013; Bobbert et al., 2017). Por lo tanto, el diseño de andamios porosos con morfología interna de SMTP es una opción viable si además se cuenta con la posibilidad de construir dichas estructuras mediante fabricación aditiva o impresión 3D utilizando ácido poliláctico (PLA) que es un poliéster alifático sintético biodegradable y biocompatible, muy utilizado en aplicaciones biomédicas (Farah, Anderson, \& Langer, 2016). De forma general, las SMTP exhiben las mejores propiedades requeridas en aplicaciones biomédicas. Por ejemplo las estructuras P y G muestran los mayores valores de permeabilidad a fluido y distribución de los esfuerzos bajo carga axial entre las estructuras con simetría cúbica para los mismos valores de tamaño de poros y porosidad (Blanquer et al., 2017; Jung \& Torquato, 2005; Melchels et al., 2010). Por otro lado hay estudios experimentales y computacionales que concluyen que la estructura $\mathrm{P}$ es la que exhibe las mejores propiedades mecánicas de 
rigidez y resistencia (Maskery et al., 2017; Montazerian, Davoodi, Asadi-Eydivand, Kadkhodapour, \& Solati-Hashjin, 2017; Restrepo, Ocampo, Ramírez, Paucar, \& García, 2017).

En la fase de diseño del andamio se pueden combinar dos o más de estas estructuras logrando una transición más o menos continua de la morfología (porosidad, tamaño de poros y distribución de estos) en la nueva estructura híbrida. La hibridación puede lograrse mediante la combinación de las ecuaciones implicitas de cada superficie a partir de un código en Mathematica (Yang, Quan, Zhang, \& Tian, 2014; Yang \& Zhou, 2014) utilizando la función sigmoidea, lo que permite variar el tamaño de poros y la porosidad mediante la variación de las constantes de diseño en cada ecuación para cada estructura SMTP individualmente. Por lo tanto es de interés investigar cómo influyen los parámetros de diseño de cada estructura por separado en las propiedades morfológicas de la estructura híbrida.

El objetivo de este trabajo es estudiar la relación funcional entre las constantes de diseño de las estructuras P y G y las propiedades morfológicas de ellas (porosidad y tamaño de poros) y de estructuras híbridas prismáticas y cilíndricas obtenidas por diseño computacional mediante la función sigmoidea. El artículo se divide en tres secciones: Introducción, Materiales y Métodos, donde se describen el equipamiento y los procedimientos empleados en esta investigación y Análisis de Resultados donde se presenta el análisis de regresión multilineal con la discusión de la pertinencia de los modelos bilineales para caracterizar las propiedades morfológicas de las estructuras híbridas. Finalmente se muestran las conclusiones del trabajo y se trazan las líneas de las futuras investigaciones en la temática.

\section{Marco Teórico}

\section{Ecuaciones}

En la Tabla 1 se presentan los parámetros de las estructuras $\mathrm{P}$ y $\mathrm{G}$ donde $X=n_{x} \pi x, Y=n_{y} \pi y, Z=n_{z} \pi z$. Los valores de $\left(n_{x}, n_{y}, n_{z}\right)$ permiten manipular el tamaño de la celda unitaria y por lo tanto el tamaño de poros en cada dirección de los ejes coordenados, mientras que el parámetro C (CP y CG) controla la porosidad.

Tabla 1.

Datos de las SMTP Giroide (G) y P de Schwarz.

\begin{tabular}{|c|c|}
\hline SMTP & Características \\
\hline $\mathbf{P}$ & $\begin{array}{l}\text { Nombre: Primitiva de Schwarz } \\
\text { Ecuación: } \phi_{1}=\cos (X)+\cos (Y)+\cos (Z)=C P \\
\text { Tipo de simetría: cúbica }(\mathrm{BCC})\end{array}$ \\
\hline $\mathbf{G}$ & $\begin{array}{l}\text { Nombre: Giroide } \\
\text { Ecuación: } \phi_{2}=\cos (X) \sin (Y)+\cos (Y) \sin (Z)+\cos (Z) \sin (X)=C G \\
\text { Tipo de Simetría: cúbica }(\mathrm{BCC})\end{array}$ \\
\hline
\end{tabular}

De manera general, valores grandes de $\left(n_{x}, n_{y}, n_{z}\right)$ resultan en menores tamaños de poros sin afectar directamente la porosidad, mientras que la porosidad se incrementa linealmente con $\mathrm{C}$, siendo este el único parámetro que la afecta. En estas ecuaciones $\phi_{\text {hib }} \geq 0$ representa el sólido mientras que $\phi_{\text {hib }} \leq 0$ representa el espacio ocupado por los poros. La estructura híbrida se define como:

$$
\phi_{\text {hib }}=\alpha(k, z) \phi_{1}+\alpha(-k, z) \phi_{2} \geq 0
$$

\section{Función de enlace}

En la Figura 1 se presenta la función de enlace sigmoidea $\alpha(k, z) \in[0,1]$ para varios valores del parámetro $\mathrm{k}$ (parámetro de enlace), donde notamos en línea de rayas y puntos el valor $\mathrm{k}=0.5$ que es recomendado en la literatura (Yang et al., 2014). Esta función actúa como puente para conectar de manera suave y continua a dos morfologías diferentes como la $\mathrm{P}$ y la $\mathrm{G}$. El método de la función sigmoidea constituye una manera eficiente de conectar dos estructuras en una híbrida e incluso puede utilizarse de forma recursiva para conectar varias estructuras y de esta forma cambiar la morfología final de poros (Yang et al., 2014). La 


\section{ORANGE JOURNAL}

función $B(x, y, z)=0$ define una frontera entre dos estructuras que, de acuerdo con la literatura, puede ser un cilindro, esfera o plano (Yang et al., 2014). Para el diseño de las estructuras prismáticas se utiliza $B(x, y, z)=z-z_{0}$ que define un plano perpendicular al eje $\mathrm{Z}$ (caso de la figura), mientras que para las probetas cilíndricas $B(x, y, z)=r^{2}-\left(x^{2}+y^{2}\right)$ que define un cilindro con eje longitudinal en $Z$. Esto permite la unión de dos estructuras prismáticas a lo largo del eje Z (mayor longitud) y la unión de dos estructuras distribuidas radialmente en una estructura cilíndrica.

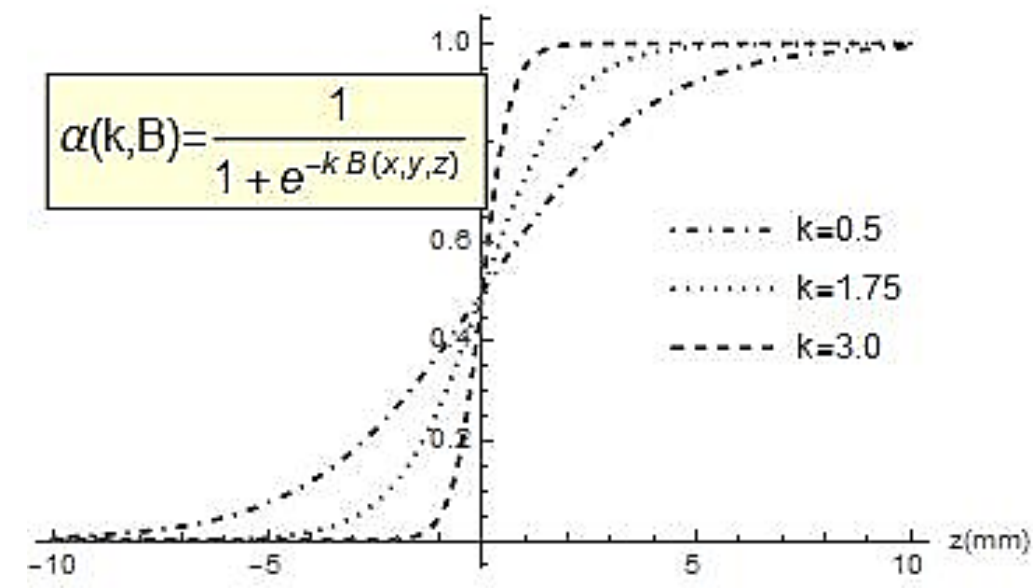

Figura 1. Función de enlace sigmoidea

\section{Metodología}

Esta es una investigación teórica que busca determinar la relación funcional entre los factores de diseño CP y CG con propiedades morfológicas de las estructuras como la porosidad y el tamaño de poros. En el siguiente epígrafe se describe el diseño de experimentos empleado en esta investigación y los procedimientos y métodos que se emplearon para la determinación de las propiedades morfológicas mencionadas.

\section{Diseño de experimentos}

En el diseño de las estructuras $\mathrm{G}$ y $\mathrm{P}$ se toma al parámetro $\mathrm{C}$ como factor en un esquema de 5 niveles para determinar las ecuaciones de porosidad y tamaño de poros. En el caso de las estructuras híbridas se propone un diseño factorial $3^{2}$ tomando como factores la constante C de cada SMTP que se denominaron como CG y CP. Las variables de respuesta son la porosidad (Por) medida en porciento y el tamaño de poros (TP) en micrómetros. El diseño elegido responde a la hipótesis de que no existe interacción entre las variables independientes pues cada una de ellas está relacionada con una SMTP diferente. Los niveles de análisis de las variables se presentan en la Tabla 2 para cada estructura por separado y en la Tabla 3 para las estructuras híbridas. El procesamiento estadístico para determinar la relación entre porosidad y tamaño de poros con los factores CP y CG se realizó con Statgraphics 18-X64 y utilizando las funciones de procesamiento estadístico de Mathematica.

Tabla 2.

Niveles de factores para $P$ y $G$ por separado.

\begin{tabular}{|c|c|c|}
\hline Nro & CP & CG \\
\hline $\mathbf{1}$ & -0.5 & -0.7 \\
\hline $\mathbf{2}$ & -0.225 & -0.575 \\
\hline $\mathbf{3}$ & 0.05 & -0.45 \\
\hline $\mathbf{4}$ & 0.325 & -0.325 \\
\hline $\mathbf{5}$ & 0.6 & -0.2 \\
\hline
\end{tabular}


Tabla 3.

Niveles de factores para estructuras híbridas GP prismáticas y cilíndricas.

\begin{tabular}{|c|c|c|}
\hline Nro Exp & CP & CG \\
\hline $\mathbf{1}$ & -0.5 & -0.7 \\
\hline $\mathbf{2}$ & 0.05 & -0.7 \\
\hline $\mathbf{3}$ & 0.6 & -0.7 \\
\hline $\mathbf{4}$ & -0.5 & -0.45 \\
\hline $\mathbf{5}$ & 0.05 & -0.45 \\
\hline $\mathbf{6}$ & 0.6 & -0.45 \\
\hline $\mathbf{7}$ & -0.5 & -0.2 \\
\hline $\mathbf{9}$ & 0.05 & -0.2 \\
\hline
\end{tabular}

\section{Normas del diseño computacional}

Las probetas fueron diseñadas utilizando el software CAS Wolfram Mathematica v11.2 a partir de un código propio y sus dimensiones son de $12.7 \times 12.7 \times 25.4 \mathrm{~mm}$ de acuerdo con la norma ASTM D695_15 (ASTM-International, 2015) para las probetas prismáticas, mientras que las probetas cilíndricas se diseñaron de $15 \mathrm{~mm}$ de radio y $10 \mathrm{~mm}$ de altura. El objeto 3D generado en el software es luego exportado a formato STL y abierto en AutoDesk Meshmixer v3.4.474 (Autodesk, Inc 2017) para ser analizado y corregido debido a posibles errores de mallado. El STL final es obtenido una vez que pasa la prueba de corrección en Meshmixer.

Para lograr un tamaño de poros adecuado en el rango del hueso trabecular $(100-900 \mu \mathrm{m})$ la celda unitaria debe medir entre 2 y $3 \mathrm{~mm}$. Además, el diseño debe tener en cuenta la periodicidad espacial de estas estructuras ya que una cantidad de celdas par o impar puede afectar la calidad de la impresión, por lo que elegimos una cantidad de celdas de $6 \times 6 \times 12$ para las probetas prismáticas lo que nos da un tamaño de celda unitaria de aproximadamente $2.12 \mathrm{~mm}$ de acuerdo con:

$$
\text { Tamaño } C U=\frac{\text { Dimensiones de probeta }}{\text { Cantidad de celdas }}
$$

En el caso de las probetas cilíndricas se utilizó un tamaño de celdas de $2.3 \mathrm{~mm}$. La calidad del STL generado a partir del diseño se controla en el software con la opción PlotPoints $\rightarrow 120$ de la función RegionPlot3D que es la que genera la superficie implícita, aunque no es la única función en Mathematica capaz de generar las estructuras en 3D.

\section{Caracterización microestructural}

La caracterización microestructural comprende la determinación de la porosidad y el tamaño de poros en la fase de diseño. La porosidad se determina analizando el STL de la probeta con la herramienta Analysis $\rightarrow$ Stability de Meshmixer que permite determinar el volumen de sólido y el área superficial. El volumen total teórico será largo $\times$ ancho $\times$ altura para las probetas prismáticas y $\pi r^{2} h$ para las probetas cilíndricas, por lo que la porosidad teórica será:

$$
\phi_{T e o}=\left(1-\frac{V_{\text {Sólido }}}{V_{\text {Teo }}}\right) \times 100 \%
$$

Para determinar el tamaño de poros, se realiza una foto mediante captura de pantalla del STL abierto en Meshmixer y la imagen en JPG es pre-procesada en Microsoft Paint para obtener la región de interés. Esta imagen es luego procesada por un código propio creado en Mathematica que utiliza un algoritmo de segmentación por inundación (Watershed) para seleccionar las áreas de poros y separarlas del fondo de la imagen. Mediante la función ComponentMeasurements de Mathematica es posible realizar una serie de cálculos sobre los objetos segmentados que permiten determinar los estimados del tamaño de poros. 


\section{ORANGE JOURNAL}

\section{Resultados}

En la Tabla 4 aparecen los resultados de porosidad y tamaño de poros para cada una de las estructuras P y $\mathrm{G}$ para los 5 niveles. Con estos datos, los resultados de regresión para la porosidad y el tamaño de poros son los de la Tabla 5.

Tabla 4.

Resultados del experimento para las estructuras $P$ y G por separado.

\begin{tabular}{|c|c|c|c|c|c|c|}
\hline Nro & $\mathbf{C P}$ & $\phi_{P}[\mathbf{\%}]$ & $\mathbf{T P}_{\mathbf{P}}[\boldsymbol{\mu m}]$ & $\mathbf{C G}$ & $\boldsymbol{\phi}_{G}[\boldsymbol{\%}]$ & $\mathbf{T P}_{\mathbf{G}}[\boldsymbol{\mu m}]$ \\
\hline $\mathbf{1}$ & -0.5 & 64.41 & 1253.39 & -0.7 & 73.07 & 782.04 \\
\hline $\mathbf{2}$ & -0.225 & 56.48 & 1088.94 & -0.575 & 68.87 & 715.593 \\
\hline $\mathbf{3}$ & 0.05 & 48.56 & 916.198 & -0.45 & 64.72 & 652.146 \\
\hline $\mathbf{4}$ & 0.325 & 40.64 & 741.232 & -0.325 & 60.61 & 594.606 \\
\hline $\mathbf{5}$ & 0.6 & 32.69 & 534.964 & -0.2 & 56.52 & 530.834 \\
\hline
\end{tabular}

Tabla 5.

Resultados de regresión para porosidad y tamaño de poros para $P$ y $G$.

\begin{tabular}{|c|c|c|c|c|c|c|}
\hline \multicolumn{7}{|c|}{ ESTRUCTURA P } \\
\hline & \multicolumn{3}{|c|}{ POROSIDAD } & \multicolumn{3}{|c|}{ TAMAÑO DE POROS } \\
\hline & Estimado & $\begin{array}{l}\text { Error } \\
\text { Estand }\end{array}$ & Valor-p & Estimado & $\begin{array}{l}\text { Error } \\
\text { Estand }\end{array}$ & Valor-p \\
\hline Constante & 49.9981 & 0.0041786 & $\begin{array}{l}1.287 \\
\times 10^{-12}\end{array}$ & 939.391 & 6.3615 & $\begin{array}{l}6.848 \\
\times 10^{-7}\end{array}$ \\
\hline \multirow[t]{4}{*}{$\mathbf{C P}$} & -28.8285 & 0.0106567 & $\begin{array}{l}1.114 \\
\times 10^{-10}\end{array}$ & -648.931 & 16.2236 & $\begin{array}{l}3.438 \\
\times 10^{-5}\end{array}$ \\
\hline & \multicolumn{3}{|c|}{$R^{2}=1.00$} & \multicolumn{3}{|c|}{$R^{2}=0.998128$} \\
\hline & \multicolumn{3}{|c|}{$R_{\text {ajust }}^{2}=0.99999$} & \multicolumn{3}{|c|}{$R_{\text {ajust }}^{2}=0.997505$} \\
\hline & \multicolumn{3}{|c|}{ Ecuación: $\phi_{P}=49.9981-28.8285 * C P$} & \multicolumn{3}{|c|}{$\begin{array}{c}\text { Ecuación: } T P_{P}=939.391-648.931 * \\
C P\end{array}$} \\
\hline \multicolumn{7}{|c|}{ ESTRUCTURA G } \\
\hline & Estimado & $\begin{array}{l}\text { Error } \\
\text { Estand }\end{array}$ & Valor-p & Estimado & $\begin{array}{l}\text { Error } \\
\text { Estand }\end{array}$ & Valor-p \\
\hline Constante & 49.8722 & 0.0457117 & $1.698 \times 10^{-9}$ & 430.62 & 3.22125 & $\begin{array}{l}9.229 \\
\times 10^{-7}\end{array}$ \\
\hline \multirow[t]{4}{*}{ CG } & -33.0813 & 0.0945477 & $5.148 \times 10^{-8}$ & -498.719 & 6.66268 & $\begin{array}{l}5.255 \\
\times 10^{-6}\end{array}$ \\
\hline & \multicolumn{3}{|c|}{$R^{2}=0.999975$} & \multicolumn{3}{|c|}{$R^{2}=0.999465$} \\
\hline & \multicolumn{3}{|c|}{$R_{a j u s t}^{2}=0.999967$} & \multicolumn{3}{|c|}{$R_{\text {ajust }}^{2}=0.999286$} \\
\hline & \multicolumn{3}{|c|}{ Ecuación: $\phi_{G}=49.8722-33.0813 * C G$} & \multicolumn{3}{|c|}{$\begin{aligned} \text { Ecuación: } T P_{G}= & 430.62-498.719 * \\
& C G\end{aligned}$} \\
\hline
\end{tabular}

En la Tabla 6 se exponen los resultados de los experimentos de las estructuras híbridas GP prismáticas y cilíndricas. El diseño factorial utilizado permite disponer de mayor cantidad de datos para estimar los efectos de interacción entre los factores y de esta forma establecer el mejor modelo que correlaciona las variables de respuesta de porosidad y tamaño de poros con los factores $\mathrm{CP}$ y $\mathrm{CG}$, dado que en esta fase de diseño no se realizan réplicas experimentales. 
Tabla 6.

Resultados del experimento para las estructuras GP prismáticas y cilíndricas.

\begin{tabular}{|c|c|c|c|c|c|c|}
\hline & & \multicolumn{1}{|c|}{} & & \\
\hline Nro Exp & CP & CG & $\phi_{\text {Pris }}[\%]$ & TP $P_{\text {Pris }}[\mu m]$ & $\phi_{\text {Cil }}[\%]$ & TP $P_{\text {Cil }}[\mu m]$ \\
\hline $\mathbf{1}$ & -0.5 & -0.7 & 73.14 & 1373.63 & 64.47 & 1065.57 \\
\hline $\mathbf{2}$ & 0.05 & -0.7 & 65.45 & 1238.4 & 51.98 & 863.957 \\
\hline $\mathbf{3}$ & 0.6 & -0.7 & 57.20 & 1029.03 & 39.74 & 613.023 \\
\hline $\mathbf{4}$ & -0.5 & -0.45 & 68.21 & 1206.61 & 62.67 & 1015.05 \\
\hline $\mathbf{5}$ & 0.05 & -0.45 & 60.41 & 1071.58 & 50.16 & 808.153 \\
\hline $\mathbf{6}$ & 0.6 & -0.45 & 51.88 & 911.436 & 37.92 & 561.275 \\
\hline $\mathbf{7}$ & -0.5 & -0.2 & 63.26 & 1041.06 & 60.85 & 977.061 \\
\hline $\mathbf{8}$ & 0.05 & -0.2 & 55.18 & 900.075 & 48.34 & 745.64 \\
\hline $\mathbf{9}$ & 0.6 & -0.2 & 46.66 & 802.982 & 36.09 & 494.389 \\
\hline
\end{tabular}

Por otra parte, en la Tabla 7 se observan los resultados más importantes de la regresión multilineal para la porosidad y el tamaño de poros en las probetas prismáticas y cilíndricas sin tener en cuenta los efectos de interacción.

Tabla 7.

Resultados de regresión para las estructuras híbridas prismáticas y cilíndricas.

\begin{tabular}{|c|c|}
\hline \multicolumn{2}{|c|}{ PROBETAS PRISMÁTICAS } \\
\hline POROSIDAD & TAMAÑO DE POROS \\
\hline $\begin{array}{l}\text { Ecuación: } \phi_{\text {Pris }}=51.6896-14.8068 * C P- \\
20.4535 * C G\end{array}$ & $\begin{array}{l}\text { Ecuación: } T P_{P r i s}=808.085-266.016 * C P- \\
597.962 * C G\end{array}$ \\
\hline$R^{2}=0.999486$ & $R^{2}=0.984305$ \\
\hline$R_{\text {ajust }}^{2}=0.999315$ & $R_{\text {ajust }}^{2}=0.979074$ \\
\hline \multicolumn{2}{|c|}{ PROBETAS CILÍNDRICAS } \\
\hline $\begin{array}{l}\text { Ecuación: } \phi_{C i l}=48.0986-22.498 * C P- \\
7.2726 * C G\end{array}$ & $\begin{array}{l}\text { Ecuación: } T P_{C i l}=717.22-420.907 * C P- \\
216.937 * C G\end{array}$ \\
\hline$R^{2}=0.999965$ & $R^{2}=0.996962$ \\
\hline$R_{\text {ajust }}^{2}=0.999954$ & $R_{\text {ajust }}^{2}=0.99595$ \\
\hline
\end{tabular}

\section{Discusión de resultados}

\section{Resultados de la segmentación con Mathematica}

El software Mathematica nos permite diseñar las estructuras mediante la función RegionPlot3D y realizar el análisis de segmentación de las fotos mediante un algoritmo de inundación (Watershed) para determinar el tamaño de poros. En la Figura 2 (a y b) se muestran una probeta prismática diseñada en Mathematica junto a la foto frontal procesada por el algoritmo de segmentación que señala en color rojo los poros de la estructura. En la Figura 2 (c y d) se muestra el mismo procesamiento para las probetas cilíndricas. 


\section{ORANGE JOURNAL}

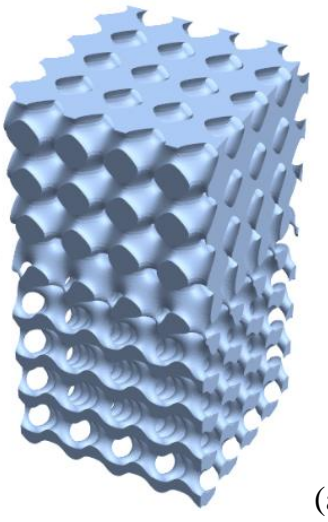

(a)

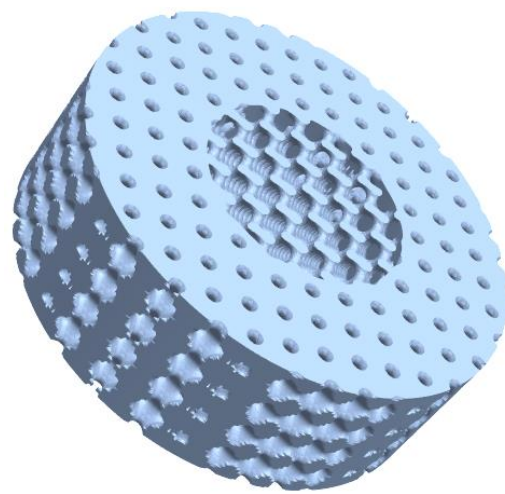

(c)

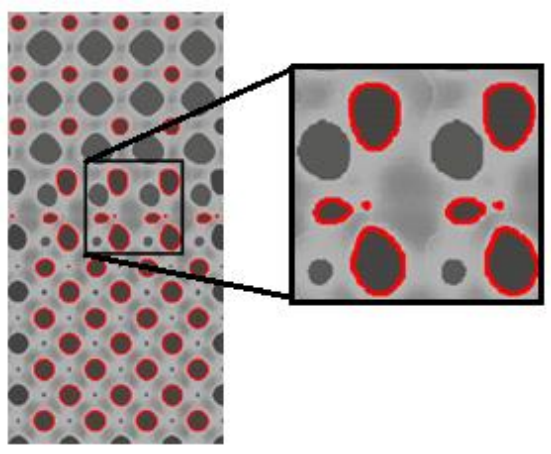

(b)

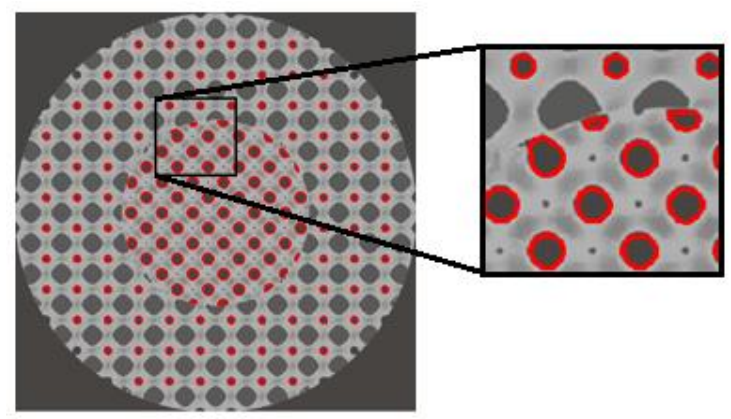

(d)

Figura 2. (a) y (b): Probeta prismática del experimento $3(\mathrm{CP}=0.6$ y $\mathrm{CG}=-0.7)$ con su correspondiente foto procesada para tamaño de poros. (c) y (d) Idem para la probeta cilíndrica del experimento 3.

Como puede notarse del detalle en la Figura 2, el algoritmo desecha aquellas partes abiertas que están en contacto con el borde. Aunque la foto aparece en colores grises, en realidad la segmentación se lleva a cabo sobre una versión binaria (negro y blanco) de cada foto para lograr el contraste adecuado para el análisis, tomando el fondo más oscuro de la figura como poros abiertos. En realidad lo que se obtiene mediante este método es un estimado de la porosidad o podríamos decir que es una porosidad superficial. No obstante como estas estructuras son totalmente conectadas, no existe porosidad oculta ni oclusiones. Además, aunque los poros en la parte frontal no tienen las mismas dimensiones que en la profundidad de la probeta, esto puede tenerse en cuenta al evaluar esta porosidad superficial en otras caras de la probeta. Un análisis más detallado necesitaría de cortes en la estructura 3D, lo cual puede ser tema de trabajos futuros.

\section{Análisis de regresión de las estructuras $P$ y $G$}

En la Tabla 5 puede observarse que para cada una de las estructuras, en cada variable de respuesta, los pvalores de los coeficientes de regresión son mucho menores que el nivel de significación del $5 \%$, lo que indica que en todos los casos estos valores son significativamente diferentes de cero para el nivel de confianza de $95 \%$ y no pueden ser eliminados del modelo. Los valores de $\mathrm{R}^{2}$ indican que el modelo lineal escogido explica más del $99 \%$ de la variabilidad en la porosidad y el tamaño de poros lo que se confirma además por los valores de $R_{\text {ajust }}^{2}$ que son muy próximos al $99 \%$ e indican una buena confiabilidad en el modelo lineal dando una concordancia casi perfecta entre los valores observados o medidos con los predichos por las ecuaciones de regresión lineal.

Otros resultados de test estadísticos realizados sobre estos datos permiten concluir que el modelo de regresión lineal escogido es apropiado para la descripción de la dependencia funcional entre porosidad y tamaño de poros con el parámetro de diseño C. Para la estructura P, el test de Durbin-Watson (González, Liste, \& Felpeto, 2013a) da valores de 2.31737 y 1.69889 para la porosidad y tamaño de poros, 
respectivamente. En la estructura $\mathrm{G}$ los resultados son de 1.44619 y 2.07531 para porosidad y tamaño de poros, respectivamente. Estos valores permiten concluir que no existe autocorrelación entre los residuos de regresión para cada variable de respuesta al nivel de significación del 95\%. Por otro lado, los test de Breusch-Pagan (González, Liste, \& Felpeto, 2013b) realizados en cada variable y para cada estructura revelan que existe homocedasticidad en los residuos de regresión (varianza constante).

Para comprobar la distribución normal de los residuos de regresión se realizaron varias pruebas estadísticas como las de Kolmogorov-Smirnov (Corder \& Foreman, 2009), Anderson-Darling (T. W. Anderson \& Darling, 1952; D’Agostino \& Stephens, 1986; Stephens, 1974) y Shapiro-Wilk (Shapiro, Wilk, \& Chen, 1968) que realizan comparaciones entre distribuciones de probabilidad tomando como referencia la distribución normal. La prueba de Kolmogorov-Smirnov tiene mayor sensibilidad para valores próximos a la mediana de la distribución, mientras que la prueba de Anderson-Darling es más sensible a valores extremos cercanos a las colas de las distribuciones y la prueba de Shapiro-Wilk es muy definitiva para muestras pequeñas $(\mathrm{n}<50)$. En todos los casos, los p-valores de cada uno de estos estimadores son mayores que el nivel de significación del $5 \%$ indicando que los residuos de regresión tienen distribución normal, lo cual es un indicativo de la pertinencia del modelo lineal en cada caso.

\section{Análisis de regresión de las estructuras híbridas GP}

En la Figura 3 podemos observar los Gráficos de Pareto Estandarizados para la porosidad y el tamaño de poros de las estructuras híbridas prismáticas y cilíndricas. En las probetas cilíndricas los p-valores del efecto de interacción $(\mathrm{AB})$ entre los factores son de 0.8422 y 0.2890 para la porosidad y el tamaño de poros respectivamente, por lo que al ser mayores que 0.05 indican que estos efectos pueden despreciarse y el modelo puede simplificarse al eliminar el término de interacción. En el caso de las probetas prismáticas esto también puede realizarse para la porosidad $(\mathrm{p}=0.137204)$ pero en el caso del tamaño de poros $\mathrm{p}=0.02288<0.05$ por lo no debería ser eliminado del modelo. Sin embargo este valor es bastante cercano a 0.05 lo que implica que el efecto de interacción es muy débil y podría eliminarse.
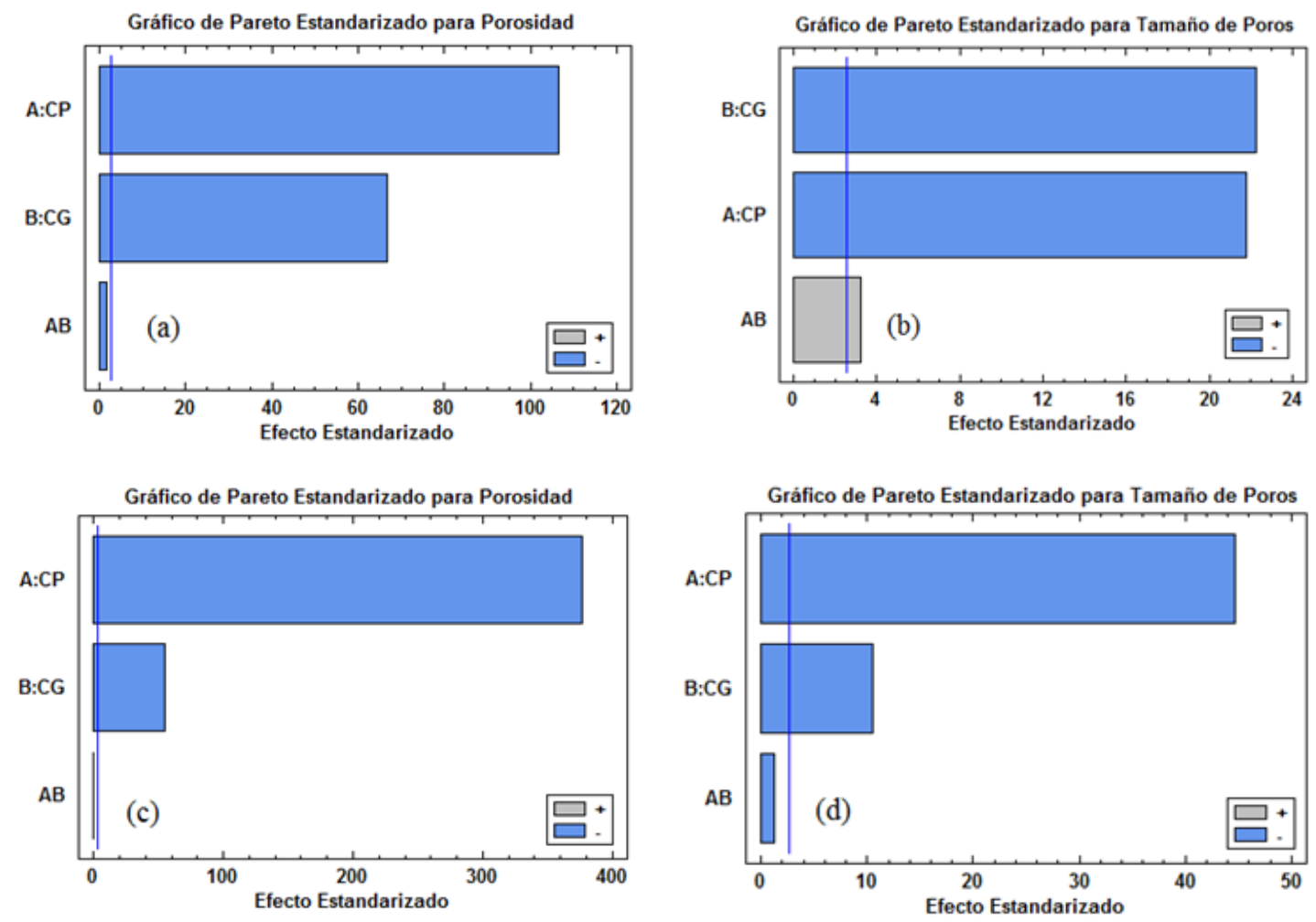

Figura 3. Gráficos de Pareto Estandarizados: (a) y (b) para la porosidad y tamaño de poros de las probetas prismáticas, (c) y (d) para las probetas cilíndricas. 
Podemos apreciar que aún cuando se eliminan los efectos de interacción en el modelo del tamaño de poros para las probetas prismáticas (caso b de la Figura 3) se tiene que $R^{2}=0.984305$ lo cual es aún muy buen indicador de linealidad en el modelo simplificado. En todos los casos, el estadístico de Durbin-Watson se obtiene con p-valores muy superiores a 0.05 lo que indica que no existe autocorrelación serial entre los residuos de regresión al $95 \%$ del nivel de confianza, lo que a su vez indica la pertinencia del modelo elegido.

\section{Conclusiones}

En el diseño de estructuras híbridas de SMTP mediante software CAS a partir de sus ecuaciones implícitas es necesario tener en cuenta la periodicidad de las SMTP por separado y controlar el tamaño de la celda unitaria de acuerdo a la precisión permitida por la impresora 3D. El software Mathematica es una opción viable para realizar este tipo de diseños dada la precisión y calidad del STL obtenido y la disponibilidad de un arsenal de funciones matemáticas para lograr los resultados.

El análisis de los datos de regresión muestra que los efectos de interacción entre los factores CG y CP, en cada modelo para las variables de respuesta de Porosidad y Tamaño de Poros, pueden ser despreciados y que en ambos casos se obtiene un modelo lineal generalizado que permite establecer una relación funcional entre los factores de diseño de cada estructura SMTP por separado y las variables de respuesta. Esto implica que los factores CG y CP que controlan la porosidad de manera lineal en cada SMTP por separado (G y P) también controlan la porosidad de forma lineal en el modelo híbrido. El tamaño de poros se controla también por los factores $C$ y por el tamaño de la celda unitaria que en este experimento se ha mantenido igual para ambas SMTP y de esta forma se obtiene también un control lineal del tamaño de poros a partir de los factores CG y CP. Los modelos obtenidos son estadísticamente significativos hasta un $95 \%$ de nivel de confianza.

\section{Referencias bibliográficas}

Ahmadi, S. M., Yavari, S. A., Wauthle, R., Pouran, B., Schrooten, J., Weinans, H., \& Zadpoor, A. A. (2015). Additively Manufactured Open-Cell Porous Biomaterials Made from Six Different SpaceFilling Unit Cells: The Mechanical and Morphological Properties. Materials, 8, 1871-1896. doi: $10.3390 / \mathrm{ma} 8041871$

Amirkhani, S., Bagheri, R., \& Zehtab Yazdi, A. (2012). Effect of pore geometry and loading direction on deformation mechanism of rapid prototyped scaffolds. Acta Materialia, 60(6), 2778-2789. doi:https://doi.org/10.1016/j.actamat.2012.01.044

Anderson, D., Davis, H., Nitsche, J., \& Scriven, L. (1990). Periodic surfaces of prescribed mean curvature. In Physics of amphiphilic layers (pp. 130-130): Springer.

Anderson, T. W., \& Darling, D. A. (1952). Asymptotic Theory of Certain "Goodness of Fit" Criteria Based on Stochastic Processes. The Annals of Mathematical Statistics (2), 193-212, 120.

Bidan, C. M., Wang, F. M., \& Dunlop, J. W. C. (2013). A three-dimensional model for tissue deposition on complex surfaces. Computer methods in biomechanics and biomedical engineering, 16(10), 1056-1070. doi:10.1080/10255842.2013.774384

Blanquer, S. B., Werner, M., Hannula, M., Sharifi, S., Lajoinie, G. P., Eglin, D., Grijpma, D. W. (2017). Surface curvature in triply-periodic minimal surface architectures as a distinct design parameter in preparing advanced tissue engineering scaffolds. BioFa, 9(2), 025001.

Bobbert, F., Lietaert, K., Eftekhari, A. A., Pouran, B., Ahmadi, S., Weinans, H., \& Zadpoor, A. (2017). Additively manufactured metallic porous biomaterials based on minimal surfaces: A unique combination of topological, mechanical, and mass transport properties. Acta Biomaterialia, 53, 572-584.

Corder, G. W., \& Foreman, D. I. (2009). Chapter 2. Testing data for normality. In Nonparametric statistics for non-statisticians: a step-by-step approach. United States: John Wiley \& Sons, Inc.

Cuan-Urquizo, E., Barocio, E., Tejada-Ortigoza, V., Pipes, R. B., Rodriguez, C. A., \& Roman-Flores, A. (2019). Characterization of the mechanical properties of FFF structures and materials: A review on the experimental, computational and theoretical approaches. Materials, 12(6), 895. 
Cuan-Urquizo, E., Yang, S., \& Bhaskar, A. (2015). Mechanical characterisation of additively manufactured material having lattice microstructure. Paper presented at the IOP Conf. Series: Materials Science and Engineering.

D’Agostino, R. B., \& Stephens, M. A. (Eds.). (1986). Goodness-of-fit techniques. United States: Marcel Dekker, Inc.

Farah, S., Anderson, D. G., \& Langer, R. (2016). Physical and mechanical properties of PLA, and their functions in widespread applications - A comprehensive review. Advanced Drug Delivery Reviews, 107, 367-392. doi:https://doi.org/10.1016/j.addr.2016.06.012

Gandy, P. J. F., Bardhan, S., Mackay, A. L., \& Klinowski, J. (2001). Nodal surface approximations to the P,G,D and I-WP triply periodic minimal surfaces. Chemical Physics Letters, 336(3), 187-195. doi:https://doi.org/10.1016/S0009-2614(00)01418-4

Giannitelli, S. M., Accoto, D., Trombetta, M., \& Rainer, A. (2014). Current trends in the design of scaffolds for computer-aided tissue engineering. Acta Biomaterialia, 10(2), 580-594. doi:https://doi.org/10.1016/j.actbio.2013.10.024

González, C. G., Liste, A. V., \& Felpeto, A. B. (2013a). Apéndice I. Tablas Estadísticas: Tabla de DurbinWatson. In Tratamiento de datos con R, STATISTICA y SPSS (pp. 875). España: Editorial Díaz de Santos.

González, C. G., Liste, A. V., \& Felpeto, A. B. (2013b). Capítulo XI. Regresiones. In Tratamiento de datos con R, STATISTICA y SPSS (pp. 460). España: Editorial Díaz de Santos.

Han, L., \& Che, S. (2018). An Overview of Materials with Triply Periodic Minimal Surfaces and Related Geometry: From Biological Structures to Self-Assembled Systems. Advanced Materials, 30(17), 1705708. doi:10.1002/adma.201705708

Hollister, S. J. (2005). Porous scaffold design for tissue engineering. Nature Materials, 4(7), 518-524. doi:10.1038/nmat1421

Jung, Y., \& Torquato, S. (2005). Fluid permeabilities of triply periodic minimal surfaces. Physical Review E, 72(5), 056319.

Kanczler, J. M., Wells, J. A., Gibbs, D. M. R., Marshall, K. M., Tang, D. K. O., \& Oreffo, R. O. C. (2020). Bone tissue engineering and bone regeneration. In R. Lanza, R. Langer, J. P. Vacanti, \& A. Atala (Eds.), Principles of Tissue Engineering (5th ed.). United States of America: Elsevier Inc.

Knychala, J., Bouropoulos, N., Catt, C., Katsamenis, O., Please, C., \& Sengers, B. (2013). Pore geometry regulates early stage human bone marrow cell tissue formation and organisation. Annals of Biomedical Engineering, 41(5), 917-930.

Lee, J. S., Cha, H. D., Shim, J. H., Jung, J. W., Kim, J. Y., \& Cho, D. W. (2012). Effect of pore architecture and stacking direction on mechanical properties of solid freeform fabrication-based scaffold for bone tissue engineering. Journal of Biomedical Materials Research Part A, 100(7), 1846-1853.

Malinauskas, M., Skliutas, E., Jonušauskas, L., Mizeras, D., Šešok, A., \& Piskarskas, A. (2015). Tailoring bulk mechanical properties of 3D printed objects of polylactic acid varying internal microarchitecture (Vol. 9505), SPIE.

Maskery, I., Sturm, L., Aremu, A. O., Panesar, A., Williams, C. B., Tuck, C. J., . . Hague, R. J. M. (2017). Insights into the mechanical properties of several triply periodic minimal surface lattice structures made by polymer additive manufacturing. Polymer. doi: 10.1016/j.polymer.2017.11.049.

Meeks III, W. H. (1990). The theory of triply periodic minimal surfaces. Indiana University Mathematics Journal, 877-936.

Melchels, F. P. W., Bertoldi, K., Gabbrielli, R., H., V. A., Feijen, J., \& Grijpma, D. W. (2010). Mathematically defined tissue engineering scaffold architectures prepared by stereolithography. Biomaterials, 31(27), 6909-6916. doi:https://doi.org/10.1016/j.actbio.2010.06.012

Montazerian, H., Davoodi, E., Asadi-Eydivand, M., Kadkhodapour, J., \& Solati-Hashjin, M. (2017). Porous scaffold internal architecture design based on minimal surfaces: A compromise between permeability and elastic properties. Materials \& Design, 126, doi: 10.1016/j.matdes.2017.04.009

Moroni, L., de Wijn, J. R., \& van Blitterswijk, C. A. (2006). 3D fiber-deposited scaffolds for tissue engineering: Influence of pores geometry and architecture on dynamic mechanical properties. Biomaterials, 27(7), 974-985. doi:https://doi.org/10.1016/j.biomaterials.2005.07.023

Naing, M. W., Chua, C. K., Leong, K. F., \& Wang, Y. (2005). Fabrication of customised scaffolds using computer-aided design and rapid prototyping techniques. Rapid Prototyping Journal, 11(4), 249259. doi: $10.1108 / 13552540510612938$ 


\section{ORANGE JOURNAL}

Nikolova, M. P., \& Chavali, M. S. (2019). Recent advances in biomaterials for 3D scaffolds: A review. Bioactive Materials, 4, 271-292. doi:https://doi.org/10.1016/j.bioactmat.2019.10.005

Norato, J. A., \& Johnson, A. J. W. (2011). A Computational and Cellular Solids Approach to the StiffnessBased Design of Bone Scaffolds. Journal of Biomechanical Engineering, 133, 091003091001:8091003-8091001. doi:10.1115/1.4004994

Restrepo, S., Ocampo, S., Ramírez, J. A., Paucar, C., \& García, C. (2017). Mechanical properties of ceramic structures based on Triply Periodic Minimal Surface (TPMS) processed by 3D printing. Journal of Physics: Conference Series, 935(012036). doi:10.1088/1742-6596/935/1/012036

Saito, E., Kang, H., Taboas, J. M., Diggs, A., Flanagan, C. L., \& Hollister, S. J. (2010). Experimental and computational characterization of designed and fabricated 50:50 PLGA porous scaffolds for human trabecular bone applications. Journal of Materials Science: Materials in Medicine, 21(8), 2371-2383. doi:10.1007/s10856-010-4091-8

Shapiro, S. S., Wilk, M. B., \& Chen, H. J. (1968). A Comparative Study of Various Tests for Normality. Journal of the American Statistical Association, 63(324), 1343-1372. doi:10.2307/2285889

Stephens, M. A. (1974). EDF Statistics for Goodness of Fit and Some Comparisons. Journal of the American Statistical Association, 69(347), 730-737. doi:10.2307/2286009

Wu, D., Spanou, A., Diez-Escudero, A., \& Persson, C. (2019). 3D-printed PLA/HA composite structures as synthetic trabecular bone: A feasibility study using Fused Deposition Modelling. Journal of the Mechanical Behavior of Biomedical Materials, 103, doi: https://doi.org/10.1016/j.jmbbm.2019.103608

Yang, N., Quan, Z., Zhang, D., \& Tian, Y. (2014). Multi-morphology transition hybridization CAD design of minimal surface porous structures for use in tissue engineering. Computer-Aided Design, 56, 11-21. doi:https://doi.org/10.1016/j.cad.2014.06.006

Yang, N., \& Zhou, K. (2014). Effective method for multi-scale gradient porous scaffold design and fabrication. Materials Science and Engineering: C, 43, 502-505. doi:https://doi.org/10.1016/j.msec.2014.07.052

Zadpoor, A. A. (2014). Bone tissue regeneration: the role of scaffold geometry. Biomaterials Science, $1-34$. 